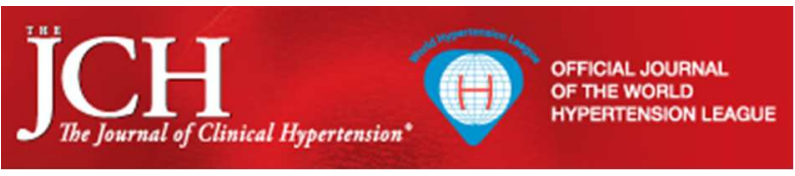

\title{
Self-reported hypertension as a public health surveillance tool: don't throw out the baby with the bathwater
}

\begin{tabular}{|r|l|}
\hline Journal: & The Journal of Clinical Hypertension \\
\hline Manuscript ID & Draft \\
\hline Diley - Manuscript type: & Letter to the Editor \\
\hline Complete List of Authors: & $\begin{array}{l}\text { Anker, Daniela; Institute of Primary Health Care (BIHAM), Research } \\
\text { Santschi, Valérie; Haute Ecole de la Sante La Source, University of Applied } \\
\text { Sciences Western Switzerland } \\
\text { Chiolero, Arnaud; Institute of Primary Health Care (BIHAM); McGill } \\
\text { University, Department of Epidemiology, Biostatistics and Occupational } \\
\text { Health }\end{array}$ \\
\hline Keywords: & $\begin{array}{l}\text { Community Based Studies, Hypertension-General, Hypertension } \\
\text { Monitoring }\end{array}$ \\
\hline Abstract: & NA \\
\hline \multicolumn{2}{|c}{} \\
\hline
\end{tabular}




\title{
Self-reported hypertension as a public health surveillance tool: don't throw out the baby with the bathwater
}

Daniela ANKER ${ }^{1}$ MSc, Valérie SANTSCHI ${ }^{2}$ PharmD PhD, Arnaud CHIOLERO ${ }^{1,3}$ MD PhD

1. Institute of Primary Health Care (BIHAM), University of Bern, Switzerland; 2. La Source, School of nursing sciences, HES-SO University of Applied Sciences and Arts of Western Switzerland, Switzerland; 3. Department of Epidemiology, Biostatistics and Occupational Health, McGill University, Montreal, Canada

\author{
Address of correspondence \\ Daniela Anker MSc \\ Institute of Primary Health Care (BIHAM) \\ Gesellschaftsstrasse 49 \\ 3012 Bern \\ Switzerland \\ Tel +41316315870 \\ Email: daniela.anker@biham.unibe.ch
}

Conflicts of interest and source of funding: The authors report no specific funding in relation to this research and no conflicts of interest to disclose

Number of words (text): 516 
Based on a thorough systematic review of epidemiological studies comparing the identification of hypertension by self-reporting with measured blood pressure, Gonçalves et al. showed that self-reported hypertension would have a low sensitivity for the identification of hypertensive individuals.1 More precisely, they showed that, on average, less than half of patients with hypertension would be identified by self-reporting. 1 Nevertheless, the author did not consider, first, that most studies based on measured blood pressure overestimate the prevalence of hypertension and, second, that self-reported hypertension entails important advantages as a public health surveillance tool.

Hypertension is a state of sustained elevated blood pressure and it is well known that an individual with elevated blood pressure at an initial visit will often have a much lower blood pressure at subsequent visits, due to habituation and regression to the mean phenomena. 2 Therefore, in practice, hypertension diagnosis is based on multiple blood pressure measurements, ideally gathered at three separated visits or more. 3 However, in most epidemiological studies, blood pressure is measured at a limited number of visits, and often at only one. For instance, in the meta-analysis of Gonçalves et al., ten studies have measured blood pressure at one visit, four at two visits, and none at three visits. 1 The way blood pressure is measured in epidemiological studies allows assessing the prevalence of elevated blood pressure, but not the prevalence of hypertension. Self-reported hypertension is less exposed to this bias because participants are asked if they are taking hypertensive drugs or if they had been diagnosed with hypertension by a physician or another healthcare professional. In both situations, we can assume that blood pressure has been measured more than once.

Of course, measuring blood pressure has unique advantages compared with self-reporting. Hence, when the goal is to identify individuals with hypertension in order to make treatment decisions, the measurement method needs to be highly sensitive and to provide accurate blood pressure estimates, and using self-reported hypertension is not conceivable.4 Studies designed 
to tackle the etiology of hypertension should also use measured blood pressure. However, when the goal is to identify prevalence and evolution of hypertension at a population level, surveys using self-report can be sufficiently informative.5 Although estimates based on selfreport can lead to an under- or overestimation of the true prevalence of hypertension, depending on age, sex, culture, education, and proximity to health care, 6 this method is simple, low-cost, and easy to apply to representative and large samples of a country.5 Further, if the degree of bias is relatively stable across time, 7 surveillance organisms can correctly assess hypertension trends over time, and draw conclusions and forecasts on evolution of hypertension and hypertension-related complications among a population.

Hence, self-reported hypertension is an imperfect proxy for the identification of hypertension and has a potential for bias. Nevertheless, it entails other important features, such as access to high shares of the population at low cost. By acknowledging the risk for bias and being aware of potential underlying causes of these biases, surveillance organisms can generate relevant estimates of hypertension trends to, in fine, guide hypertension management programs at a country or a regional level.8 


\section{References}

1. Goncalves VSS, Galvao TF, Silva MT, Kuschnir MC, Dutra ES, Carvalho KMB. Accuracy of self-reported hypertension in Brazilian adolescents: Analysis of the Study of Cardiovascular Risk in Adolescents. J Clin Hypertens (Greenwich). 2018;20(4):739-47.

2. Bovet P, Gervasoni JP, Ross AG, Mkamba M, Mtasiwa DM, Lengeler C, et al. Assessing the prevalence of hypertension in populations: are we doing it right? J Hypertens. 2003;21(3):509-17.

3. Siu AL. Screening for high blood pressure in adults: U.S. Preventive Services Task Force recommendation statement. Ann Intern Med. 2015;163(10):778-86.

4. Vargas CM, Burt VL, Gillum RF, Pamuk ER. Validity of self-reported hypertension in the National Health and Nutrition Examination Survey III, 1988-1991. Prev Med. 1997;26(5 Pt 1):678-85.

5. Guest C, Walter R, Kawachi I, Lang I, editors. Oxford Handbook of Public Health Practice. 3rd ed. Oxford University Press; 2013. P. 140-2.

6. Goldman N, Lin IF, Weinstein M, Lin YH. Evaluating the quality of self-reports of hypertension and diabetes. $J$ Clin Epidemiol. 2003;56(2):148-54.

7. Faeh D, Marques-Vidal P, Chiolero A, Bopp M. Obesity in Switzerland: do estimates depend on how body mass index has been assessed? Swiss Med Wkly. 2008;138(13-14):20410.

8. Centers for Disease Control and Prevention. Behavioral Risk Factor Surveillance System [Internet]. Atlanta, GA : Division of Population Health; [updated 2018 March 22; cited 2018 April 25]. Available from: https://www.cdc.gov/brfss/index.html. 